\title{
Isosorbide in treatment of infantile hydrocephalus
}

\author{
JOHN LORBER \\ From the Children's Hospital, Sheffield
}

\begin{abstract}
Lorber, J. (1975). Archives of Disease in Childhood, 50, 431. Isosorbide in treatment of infantile hydrocephalus. This paper reports the experiences of the second clinical trial in the use of isosorbide in the treatment of 34 selected cases of infantile hydrocephalus of all types. Subject to careful biochemical monitoring of serum electrolyte, urea, and acid-base balance, treatment with $2 \mathrm{~g} / \mathrm{kg}$ body weight 6-hourly is safe. Side effects are immediately eliminated by interrupting therapy. With lower dosage, prolonged maintenance therapy was possible, for as long as 11 months, without side effects and with need for much less frequent biochemical monitoring.

Isosorbide effectively prevented the need for shunt therapy in 10 of 34 patients ${ }^{\star}$, including 3 infants with uncomplicated congenital hydrocephalus of moderate degree and infants whose hydrocephalus was associated with spina bifida and whose cerebral mantle was between 20 to $25 \mathrm{~mm}$. In posthaemorrhagic and postmeningitic hydrocephalus valuable time was gained before shunt therapy until the infant and his CSF were fit for operation.
\end{abstract}

The high frequency and the seriousness of complications resulting from any form of shunt treatment makes this otherwise excellent method of treatment of hydrocephalus an extremely hazardous procedure. 'Revisions' may have to be carried out on numerous occasions-blockages and infections of the shunt system threaten life, vision, and intelligence. Early shunting is particularly dangerous (Lorber, 1969). In a very large series of patients whose hydrocephalus was part of the spina bifida complex there was a mortality rate of $20 \%$ within 7 years of the first operation directly attributable to shunt complications (Lorber, 1972a). These universally observed facts make it necessary to look for alternative and safer methods to treat hydrocephalus which would not necessarily be as final and practically irreversible as the present surgical techniques.

One promising drug which has emerged as a possible alternative to surgical treatment is isosorbide. This crystalline substance, 1,4:3,6-dianhydro-D-glucitol, is a derivative of mannitol. The pharmacological data about its use in experimental animals and subjects have been described (Lorber, 1973a; Shurtleff and Hayden, 1972). After a pilot trial involving 7 patients (Lorber, 1971a), a full-

Received 13 September 1974.

*Some of these might not have needed operation even without isosorbide. scale clinical study was carried out in 1971 and 1972, in which 60 patients with all types of hydrocephalus were treated with isosorbide. This study included a controlled trial in 25 infants born with spina bifida (Lorber, 1972b, 1973a).

The protocol of the first investigation arbitrarily limited the duration of treatment to at most 4 weeks. Several patients who responded satisfactorily to isosorbide relapsed after stopping therapy and according to the protocol of the investigation were operated on. The first protocol did not allow prolongation of treatment or a repetition of the course in those patients whose hydrocephalus was of such degree that operation would eventually have to be performed.

Analysis of the data from the first trial suggested that longer courses could possibly prevent the need for operation in a larger proportion of infants, and the current investigation was started in September 1972 to test this hypothesis. Further, in the first trial no infant was treated under 6 days of age. It seemed possible that by treating infants with spina bifida and hydrocephalus even earlier, progressive hydrocephalus requiring surgical treatment might be prevented.

The first investigation established that isosorbide was of no value in most infants who had severe hydrocephalus with a cerebral mantle less than 
$15 \mathrm{~mm}$ (Lorber, 1961), though occasionally even such infants temporarily benefited and time was gained until they became fit for operation, for example in hydrocephalus resulting from meningitis or intracranial haemorrhage (Lorber and Bhat, 1974). Shurtleff et al. (1973) also showed good temporary effect of isosorbide in those infants who had infected CSF and were not fit for operation.

In the first trial various dosage schedules were used. It was found that if more than $2 \mathrm{~g} / \mathrm{kg}$ body weight 4 times daily was used then biochemical complications consisting of hypernatraemia, acidosis, and rising blood urea were too common, requiring interruption of therapy-but there were no permanent after-effects and there was no fatality.

\section{Second trial}

The current trial began in September 1972. The patients included were now selected. Those who were unlikely to respond to isosorbide and were fit for operation were operated on at once. There were far fewer infants born with spina bifida available for isosorbide therapy because of the policy of selection, which was put into operation in July 1971 (Lorber, 1971b, 1972a, 1973b).

All types of infantile hydrocephalus were enrolled in this second trial. The preliminary investigations were identical to those used in the first trial, and consisted of routine air or positive contrast ventriculography, examination of CSF, biochemical examination of blood, and haematology (for details see Lorber, 1973a).

The standard starting dose of isosorbide was $2 \mathrm{~g} / \mathrm{kg}$ 6-hourly. This was shown to be the most effective dose without causing frequent or severe side effects. If this was effective then a smaller dose of $1.5 \mathrm{~g} / \mathrm{kg}$ or $1 \mathrm{~g} / \mathrm{kg}$ 6-hourly was used after 3 weeks and for as long as seemed necessary either to achieve complete arrest of hydrocephalus or until the patient was fit for operation if needed. If the response to the 'standard' dose was inadequate and if there were no side effects, then the dose was increased to $2 \mathrm{~g} / \mathrm{kg}$ 4-hourly, for a limited period. During treatment on the standard or higher dose the infant's weight, head circumference, and fontanelle tension were daily monitored; serum electrolytes, urea, and 'Astrup' data were obtained three times a week and other investigations were carried out as needed. While on standard dosage the infants remained in hospital, but if they responded well and had no side effects, then after the dose was reduced they were discharged home for maintenance therapy. Clinical and biochemical assessment were then carried out at approximately 4-weekly intervals. The duration of treatment was now dependent on the clinical response, especially the rate of increment of the head circumference and the fontanelle tension. Minor variations in the treatment schedule were practised according to day-today clinical observations.

If toxic side effects, such as hypernatraemia $\left(\mathrm{Na}^{+}\right.$ $>150 \mathrm{mEq} / \mathrm{l}$ ), or severe loss of weight, or diarrhoea and vomiting, occurred then treatment was interrupted and was resumed after recovery, usually with reduced dosage.

Between September 1972 and August 1974, 30 new cases of hydrocephalus started treatment with isosorbide. 4 others, who had been treated earlier in the first series, either had a further course or continued treatment with a more prolonged course according to the plan of the second trial. The types of patients treated, their ventriculographic findings, and the age at the time of starting therapy are given in Table I.

Judging by the experience of the first trial (Lorber, 1973a) the patients were studied in two groups. In the first group were 15 infants in whom success could be hoped for with avoidance of shunt therapy because their cerebral mantle was over $15 \mathrm{~mm}$; they did not have excessively high intracranial pressure and they did not have active meningitis or grossly abnormal CSF.

In the second group were 19 infants in whom avoidance of shunt therapy could not be expected because their cerebral mantle was $15 \mathrm{~mm}$ or less, or they had infected CSF, or extremely high pressure. It was hoped, however, that time might be gained before operation. Delay was essential in 6 infants because they had active or recent meningitis with grossly abnormal CSF. In the other 13 delay was desirable either because of gross prematurity or simply to reduce the risk of later shunt complications.

Treatment was considered to be fully successful if no operation became necessary. Partial success was achieved if operation could be delayed either by at least 28 days without causing any harm, or for a shorter period in infants with meningitis, until they and their CSF were fit for operation.

\section{Results}

Results according to anticipated response (Table II). It was anticipated that 15 patients had a good chance of avoiding operation. In 10 this expectation was fulfilled. 2 infants, both with uncomplicated congenital hydrocephalus, were treated for 11 months (Cases 16 and 10) and one, with spina bifida, for 7 months (Case 1). The others were treated for shorter periods. Follow-up after conclusion of treatment was for 1 to 2 years. With one exception they are normal, but one is profoundly handicapped through the sequelae of the myelomeningocele (Case 2). The response of 4 infants was less satisfactory, though a worthwhile delay of 29 days to 5 months was secured without harm before operation was carried out (Cases 4, 11, 25 , and 26).

Treatment was interrupted after only 4 days in one infant with posthaemorrhagic hydrocephalus, because his head continued to grow rapidly and, as he was blind and spastic, delay was likely to be dangerous. After operation he recovered his vision and at 1 year of age was normal. This baby was included among those who could have responded 
TABLE I

Isosorbide trial, September 1972 - April 1974

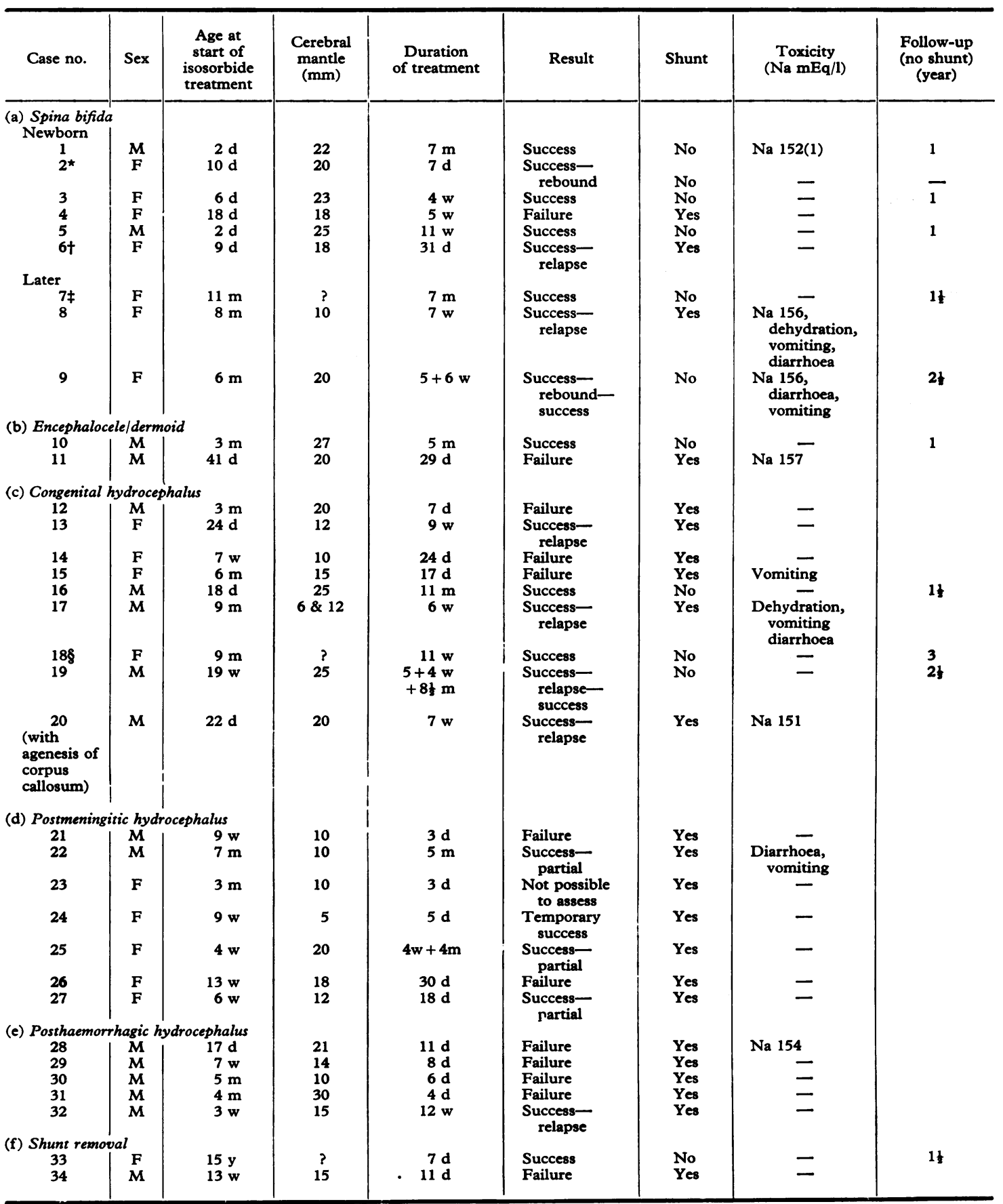

*Treatment abandoned due to severity of the spina bifida.

tHad concurrent meningitis and ventriculitis.

¥Cerebral mantle only measured at 4 months of age.

$\$$ Has also been included in the first trial.

Rebound, active hydrocephalus after stopping therapy; relapse, active hydrocephalus while still on therapy. 
TABLE II

Aims at start of treatment and results

\begin{tabular}{|c|c|c|c|c|}
\hline & No. & $\begin{array}{l}\text { Success } \\
\text { (no shunt) }\end{array}$ & $\begin{array}{c}\text { Worthwhile } \\
\text { delay }\end{array}$ & Failure \\
\hline $\begin{array}{l}\text { Arrest of hydrocephalus without shunt therapy } \\
\text { Holding hydrocephalus under control, until fit for } \\
\text { shunt therapy }\end{array}$ & 15 & $\begin{array}{r}10 \\
1\end{array}$ & $\begin{array}{l}4(\cdot 1) \\
9(7)\end{array}$ & 1 \\
\hline Total & 34 & 11 & $13(11)$ & 10 \\
\hline
\end{tabular}

^Either at least 28 days (number in parentheses) or until infant became fit for operation.

better, because his cerebral mantle was $30 \mathrm{~mm}$. However, other features, including bilateral subdural haematomata, were against a good outcome without operation (Case 31).

In the other 19 patients at best only a worthwhile delay before operation could be expected. This was achieved in 9 infants, including 2 who were treated for less than 28 days but whose CSF improved sufficiently to be ready for operation (Cases 24 and 27). The longest delay, 5 months, was achieved in 2 infants with postmeningitic hydrocephalus (Cases 22 and 25). The shunt system of a 15-yearold girl had to be removed because of septicaemia. The operation was followed by isosorbide and appropriate antibiotic treatment. Unexpectedly, she did not develop signs of intracranial hypertension after stopping isosorbide therapy and a shunt did not have to be reinserted (Case 33).

There was an interesting group of 6 infants whose initial response was excellent, but whose hydrocephalus got out of control during the latter part of isosorbide therapy, either on the full dose (2 infants) or when the dose was reduced to $1 \mathrm{~g} / \mathrm{kg} 4$ times daily. All were operated on, but valuable delay of 5 to 12 weeks was achieved before operation.

Results according to type and grade of hydrocephalus (in the larger subgroups) (Table I).

Nine infants with spina bifida. There were 6 newborn infants between 2 and 18 days of age whose cerebral mantle was between 18 and $25 \mathrm{~mm}$. The 4 with a cerebral mantle of $20-25 \mathrm{~mm}$ responded and are well, with normal milestones of development at $1-1 \frac{1}{2}$ years of age. In the other 2 , whose cerebral mantle was $18 \mathrm{~mm}$ (Cases 4 and 6), the reduction in intracranial tension was insufficient. They were operated on after 4 and 5 weeks.

Three older infants with spina bifida all responded, but one required 2 courses (Case 9) and the other was operated on after 7 weeks of treatment because on the standard dose she was hypernatraemic and dehydrated (Case 8 ).

Nine infants with uncomplicated congenital hydrocephalus. There were 9 infants with a cerebral mantle of 6-25 mm. 3 failed to respond and were operated on. 4 others responded well, but 3 relapsed while on treatment and were operated on. These included an infant with agenesis of the corpus callosum, whose lateral ventricles were not grossly dilated (mantle $20 \mathrm{~mm}$ ) but whose third ventricle was extremely big (Case 20). There remain 3 children without a shunt: those with the least severe hydrocephalus. One infant who relapsed after the first and second course had a third long course with good response. This time no rebound occurred after stopping therapy (Case 19). Another made normal progress during 11 months of treatment on a slowly reduced dosage schedule and at 18 months of age he is a normal boy (Case 16). The third had a cerebral mantle of $35 \mathrm{~mm}$ at 4 months of age. She was observed without treatment for 5 more months. Her head circumference gradually diverged above the 90th centile line, so at 9 months of age isosorbide treatment was started. The air ventriculogram was not repeated, so the thickness of the cerebral mantle was not known at this stage. She responded to treatment for 11 weeks and there was no rebound. At 3 years of age she is a normal girl (Case 18).

Seven infants with postmeningitic hydrocephalus. 7 infants were treated for postmeningitic hydrocephalus. Another infant with spina bifida (Case 6) was dealt with in that section. All were eventually operated on, but a worthwhile delay was achieved in 4. No infant in this group had a cerebral mantle over $20 \mathrm{~mm}$.

Five infants with posthaemorrhagic hydrocephalus. 5 infants were treated for posthaemorrhagic hydrocephalus. Treatment failed in 4 but all 5 
have been operated on. The infant who responded well was a premature baby who had a large number of fits from the first day of life. In the first 16 days of life his head circumference increased by $45 \mathrm{~mm}$ (normal: $12 \mathrm{~mm}$ ). On the 16th day his CSF was deeply xanthochromic and his cerebral mantle was $15 \mathrm{~mm}$. On the standard dose of isosorbide his head circumference only increased by $17 \mathrm{~mm}$ in 4 weeks (normal rate). Then, on half dose his head grew only slightly above the normal rate for $\mathbf{4}$ more weeks. He then developed acute gastroenteritis and though he recovered quickly, continuation of isosorbide in the same dose was ineffective and in the next 24 days (while at home) his head circumference increased by $45 \mathrm{~mm}$ ( 4 times normal rate). At this stage he was operated on and he made an uneventful recovery (Case 32 ).

Two other groups of encephalocele/dermoid ( 2 cases) and shunt removal ( 2 cases) are shown in Table I.

\section{Toxicity}

Few infants liked to take the isosorbide, especially during the earliest stage of the treatment, but most got used to it. Initially, treatment often had to be given by gastric tube to ensure that they received the full dose. The bulk of the solution is a problem: $1 \mathrm{~g}$ isosorbide is dissolved in $2.2 \mathrm{ml}$ liquid. This means that on the standard regimen a single dose for a $5 \mathrm{~kg}$ infant was $22 \mathrm{ml}$.

Infants were slightly unwell during treatment on the standard dose but major side effects were not common. In 24 infants no clinical or biochemical side effects were present which required even temporarily stopping therapy. Hypernatraemia of 151 to $158 \mathrm{mEq} / 1$ developed in 6 infants, while serious vomiting, diarrhoea, loss of weight, or acidosis, singly or in combination occurred less often (Table III). Stopping of therapy led to

\section{TABLE III}

\begin{tabular}{lr}
\multicolumn{2}{c}{ Toxicity } \\
\hline None & 24 \\
Hypernatraemia & 6 \\
Acidosis & - \\
Loss of weight & 2 \\
Vomiting & 3 \\
Diarrhoea & 5 \\
Other & 1 \\
\hline
\end{tabular}

immediate recovery in all, and treatment if necessary was usually continued with reduced dose without further trouble. There were no deaths or permanent side effects.

\section{Follow-up on patients in first trial}

Analysis of the results of the first series showed that 12 of the 60 isosorbide-treated patients as well as 1 'control' case of myelomeningocele survived without a shunt. Subsequently, 2 patients were operated on soon after the report was prepared. One was an infant with myelomeningocele, who is also included in the present series (Case 8), and another who had had her shunt removed because of colonization had to have it reinstated several months later. This leaves 10 treated and one 'control' child still without need for a shunt. 3 (Cases 9, 18, and 19) are included in the present series because they had further or more prolonged courses. The other 7 earlier treated children included a 9-year-old girl who is well, whose shunt did not have to be replaced after she recovered from Klebsiella meningitis, and 6 who never had a shunt. They were 2 to 3 years of age when last seen. The head circumference was below the 90th centile in 4; and 1 and $4 \mathrm{~cm}$, respectively, above the 90th centile in the other 2. The child with the biggest head circumference of $55.5 \mathrm{~cm}$ is intellectually fully normal. His head stopped growing in the past year and he shows no clinical appearance of hydrocephalus. All 6 are of normal intelligence. The only unoperated 'control' child's head is of normal size but she is dull.

\section{Discussion}

This investigation confirmed the findings of the first trial that infants with moderate hydrocephalus usually respond well to isosorbide. It is probable that a substantially larger proportion can be managed without operation with the use of isosorbide. Unfortunately, the numbers in this trial do not justify treating the results with statistical tests of significance. Nevertheless, only 2 out of $8(25 \%)$ infants with spina bifida and a cerebral mantle of 16-25 $\mathrm{mm}$ required shunt therapy, whereas before the use of isosorbide, in a much larger series, $66 \%$ of 132 infants with this degree of hydrocephalus had to be operated on (Lorber, 1971a). It was exceptional in the past that infants with uncomplicated congenital hydrocephalus of any degree could be left without treatment (Lorber and Zachary, 1968), while in this series 3 out of 9 are doing well, without operation, after stopping isosorbide therapy. The results are less satisfactory in cases of postmeningitic and posthaemorrhagic hydrocephalus, and all 12 were eventually operated on. In these infants, however, operation could often be deferred until the patient was fit for operation.

It is obvious that isosorbide will not replace shunt 
therapy for the majority of patients, except those who have uncomplicated infantile hydrocephalus with a cerebral mantle of $20 \mathrm{~mm}$ or more, and those infants with spina bifida whose cerebral mantle is at least between 15 and $25 \mathrm{~mm}$, especially if treatment is started within a few days of closure of the lesion. For spina bifida infants with lesser degrees of hydrocephalus neither medical nor surgical treatment is advised, at least until observation shows that treatment becomes necessary. Past experience with very large numbers shows that only one-third of such babies will need treatment at a later stage (Lorber, 1971a), and they are likely to respond to isosorbide.

Isosorbide is not as effective as operation and does not produce results quickly, but it is far safer. Failure or toxic symptoms can always be followed by operation whereas failure and the complications of surgery are far more dangerous. Surgery is usually irreversible.

Isosorbide is a valuable drug to deal with some cases of hydrocephalus resulting from infection or haemorrhage until the infant is fit for operation. Unfortunately, isosorbide is still only available for clinical trial, so at present babies can only be treated with it in very few centres.

Isosorbide was supplied as Hydronol by Stuart Pharmaceuticals, Division of ICI America Inc., Wilmington, Delaware, 19899, U.S.A.

\section{REFERENCES}

Lorber, J. (1961). Systematic ventriculographic studies in infants born with meningomyelocele and encephalocele. The incidence and development of hydrocephalus. Archives of Disease in Childhood, 36, 381.

Lorber, J. (1969). Ventriculo-cardiac shunts in the first week of life: results of a controlled trial in the treatment of hydrocephalus in infants born with spina bifida cystica or cranium bifidum. Developmental Medicine and Child Neurology, 11, (Suppl. 20), 13.

Lorber, J. (1971a). Medical and surgical aspects in the treatment of congenital hydrocephalus. Neuropaediatrie, 2, 239.

Lorber, J. (1971b). Results of treatment of myelomeningocele. An analysis of 524 unselected cases, with special reference to possible selection for treatment. Developmental Medicine and Child Neurology, 13, 279.

Lorber, J. (1972a). Spina bifida cystica. Results of treatment of 270 consecutive cases with criteria for selection for the future. Archives of Disease in Childhood, 47, 854.

Lorber, J. (1972b). The use of isosorbide in the treatment of hydrocephalus. Developmental Medicine and Child Neurology, 14, (Suppl. 27), 87.

Lorber, J. (1973a). Isosorbide in the medical treatment of infantile hydrocephalus. Journal of Neurosurgery, 39, 702.

Lorber, J. (1973b). Early results of selective treatment of spina bifida cystica. British Medical, fournal, 4, 201.

Lorber, J., and Bhat, U. S. (1974). Post-haemorrhagic hydrocephalus. Diagnosis, differential diagnosis, treatment, and long-term results. Archives of Disease in Childhood, 49, 751.

Lorber, J. and Zachary, R. B. (1968). Primary congenital hydrocephalus. Archives of Disease in Childhood, 43, 516.

Shurtleff, D. B. and Hayden, P. W. (1972). The treatment of hydrocephalus with isosorbide, an oral hyperosmotic agent. fournal of Clinical Pharmacology, 12, 108.

Shurtleff, D. B., Hayden, P. W., Weeks, R., and Laurence, K. M (1973). Temporary treatment of hydrocephalus and myelodysplasia with isosorbide: preliminary report. Fournal of Pediatrics, 83, 651 .

Correspondence to Dr. J. Lorber, The Children's Hospital, Sheffield S10 2TH. 